Relations industrielles

Industrial Relations

\title{
How to Manage by Objectives, by John W. Humble, New York, Amacon, 1972, 150 pp.
}

\section{Laurent Bélanger}

Volume 30, numéro 1, 1975

URI : https://id.erudit.org/iderudit/028597ar

DOI : https://doi.org/10.7202/028597ar

Aller au sommaire du numéro

Éditeur(s)

Département des relations industrielles de l'Université Laval

ISSN

0034-379X (imprimé)

1703-8138 (numérique)

Découvrir la revue

Citer ce compte rendu

Bélanger, L. (1975). Compte rendu de [How to Manage by Objectives, by John W. Humble, New York, Amacon, 1972, 150 pp.] Relations industrielles / Industrial Relations, 30(1), 127-128. https://doi.org/10.7202/028597ar

Tous droits réservés (C) Département des relations industrielles de l'Université Laval, 1975
Ce document est protégé par la loi sur le droit d'auteur. L’utilisation des services d'Érudit (y compris la reproduction) est assujettie à sa politique d'utilisation que vous pouvez consulter en ligne.

https://apropos.erudit.org/fr/usagers/politique-dutilisation/ 
d'oeuvre. Cependant, il ne faudra pas espérer y trouver les solutions miracles aux difficiles problèmes de la formation professionnelle et de son évaluation. Par contre, plusieurs leçons très utiles peuvent en être tirées.

Université Laval

Jean SEXTON

How to Manage by Objectives, by John W. Humble, New York, Amacon, 1972, 150 pp.

Ce volume est une présentation succinte d'un système intégré de management qui conserve un caractère de nouveauté pour le moment, celui de la direction par objectif ou «Management by Objectives ». L'auteur est déjà connu par ses nombreux articles et ouvrages sur le sujet et, aussi par ses projets de consultation auprès de grandes entreprises européennes et américaines. Il a voulu faire de ce dernier volume un résumé des éléments essentiels de ce «nouveau » système de management en le définissant d'abord sous un angle humaniste plutôt que technique et en signalant les écueils à éviter au moment de son introduction dans une organisation. Ceci fait l'objet de la première partie du volume. La deuxième est plutôt de caractère technique et pratique. Elle consiste, en effet, en une description des phases d'implantation de la direction par les objectifs au sein d'une organisation. L'implantation débute par un effort de planification à l'échelle de l'organisation en utilisant une démarche qui permet d'identifier d'abord les zones de résultats-clefs à l'intérieur desquels une firme peut se définir des objectifs à atteindre qui seront utilisés par la suite pour fin de contrôle c'est-à-dire, de revision des programmes d'action et du choix des ressources. Une fois les objectifs de la firme ainsi définis en termes opérationnels, il est possible de procéder à une définition des objectifs des grandes divisions, des unités administratives et de chacun des postes administratifs qui les composent. On arrive ainsi à une sorte de cascade ou hiérarchie d'objectifs à l'échelle de l'organisation. Pour chaque objectif, on trace un programme d'action et on procède à l'allocation des ressources. A chacune des étapes de réalisation, on applique des points de contrôle et la période de réalisation se termine par une double évaluation: celle de la performance et celle du potentiel de l'administrateur. C'est ainsi que la notation des cadres prend une allure nouvelle puisqu'elle repose beaucoup plus sur la performance que sur les traits de personnalités des individus. L'évaluation de la performance suivie de l'évaluation du potentiel fournissent une information valable pour élaborer des programmes de formation et un plan de carrière qui correspondent aux besoins réels des cadres. De plus, l'évaluation de la performance permet d'élaborer une politique de rémunération qui reflète plutôt l'effort et le rendement fournis que les années de services accumulées au sein d'une organisation. Cette seconde partie cherche donc à décrire l'aspect mécanique du système. La dernière consiste uniquement en une liste de contrôle (check list) qui peut être utile au moment soit de décider de l'implantation de la direction par objectifs, soit de chercher à améliorer le fonctionnement du système. Pour une organisation qui est disposée à implanter la direction par objectifs en commençant par le sommet, i.e. la haute-direction, ce petit volume s'avère un guide précieux puisque son auteur met surtout l'accent sur la planification à l'échelle de l'entreprise. Tout en étant un guide valable, l'ouvrage comporte cependant quelques lacunes. D'abord, la définition que l'auteur donne de la direction par les objectifs s'éloigne passablement de la description qu'il en fait dans la seconde partie de son ouvrage. Pour Humble, la direction par objectifs est «un système de management qui intègre les objectifs de rentabilité de l'entreprise avec les besoins des dirigeants de fournir une contribution significative et de s'actualiser au travail » (p. 4). C'est une définition plutôt abstraite qui met l'accent sur l'aspect motivationnel de la direction par objectifs. Une définition qui en traduirait mieux les caractéristiques essentielles serait la suivante: «un système intégré de management dans lequel le subordonné partage avec son supérieur la responsabilité de la détermination et de la réalisation des objectifs, et les récompenses sanctionnant l'obtention des résultats escomptés. En plus de traduire l'aspect technique de la gestion par objectifs, cette définition fait ressortir la dimension participative de ce système de management, un au- 
tre aspect que néglige de traiter en profondeur l'auteur du présent ouvrage. Sur ce plan, l'auteur traite effectivement du travail d'équipe et de l'adoption d'une philosophie participative du management découlant de la théorie $\mathrm{Y}$ de McGregor comme autant de prérequis à l'instauration du système, mais ne s'aventure pas dans une explication du lien qui peut exister entre une phisolophie participative et la nature de la participation inhérente à la gestion par objectifs.

Une autre dimension m'apparaît aussi escamotée : celle du lien qui existe entre la motivation des dirigeants et la gestion par objectifs. On retrouve au cours d'un chapitre un parallèle entre les besoins des dirigeants et la manière dont la gestion par objectifs peut les satisfaire. Cependant, l'auteur fait peu de place dans le système qu'il présente aux aspirations personnelles des dirigeants en termes d'avancement et développement personnel, puisque la définition d'objectifs se limite aux postes administratifs et non aux individus qui en sont les titulaires.

\section{Université Laval}

Labour Relations Law, Cases-Materials and Commentary, The Labour Relations Law Casebook Group, Second Edition, Kingston, Queen's University Press, 1974, 589 pp.

La réputation de ce casebook n'est plus à faire. La première édition (1970) avait su s'attirer d'élogieux commentaires. La seconde édition en est une repensée. On notera notamment que les matières qui sous-tendent la compréhension des rapports collectifs du travail sont traités de façon différente et plus approfondie. De plus, et c'est là le principal mérite de la nouvelle édition, plusieurs sujets concernant la négociation collective ont été rajoutés ou complétés.

L'ouvrage est présenté en dix thèmes : 1) The Issues (p. 1); 2) The Emergence of Collective Bargaining as Basic Public Policy in Canadian Labour Relations (p. 7) ; 3) The Right to Join a Union (p. 43) ; 4) Status Under Collective Bargaining Legislation ( $p .46) ; 5$ ) The Establishment and Termination of Collective Bargaining Rights (p. 125);
6) The Collective Bargaining/Relationship (p. 184) ; 7) The Collective Agreement and its Administration (p. 238) ; 8) Industrial Conflict: Strikes, Picketing, Boycotts and Lockouts (p. 400) ; 9) Inter-Union Rivalry Regarding Membership and Work Jurisdiction ( $p$. 496) ; 10) The Union and Those Subject to its Authority; Internal Union Affairs (p. 524).

Chacun des grands thèmes regroupent différents sous-thèmes dont on peut trouver les intitulés à la table des matières. Cette forme de présentation rend l'ouvrage d'utilisation simple et rapide dans la mesure où l'utilisateur entend lire le sous-thème en entier. On regrette l'absence d'un index analytique dont le grand mérite est de permettre de localiser rapidement un sujet particulier qui peut être abordé à différents sous-thèmes.

Le Labour Relations Law reste essentiellement un casebook. Il n'est ni un traité, ni un précis, ni un manuel sur le droit des relations du travail. Le choix des articles de doctrinales et des espèces jurisprudentielles qui sont rapportées dans cet ouvrage semble très judicieux. Les commentaires des auteurs situent particulièrement bien le lecteur. Les références faites à la législation étaient de mise. Ce casebook ne semble avoir été conçu ni pour des fins purement pédagogiques, ni pour des fins simplement pratiques. Il tente de se situer à mi-chemin entre ces deux objectifs et y réussit fort bien.

Le groupe de travail qui a signé l'ouvrage est constitué de H.W. Arthurs, P. Weiler, B. Adell et I. Christie, G. Adams et K. Norman. Ces éminentes autorités sont, pour le lecteur, une garantie du sérieux du document que l'on ne peut que fortement suggérer aux québécois de consulter pour fin d'études de droit comparé.

\section{Université Laval}

Rodrigue BLOUIN

Eléments de sociologie hospitalière, par Paul Swertz, Paris, Le Centurion, 1974, 132 pp.

Ce petit livre, dont l'édition originale allemande date de 1970 , s'adresse spécifiquement au personnel hospitalier. Il se veut une introduction, d'accès facile, à une analyse sociologique de l'hôpital. 